\title{
Three-body Force Effects on the Longitudinal Response Function of ${ }^{4} \mathrm{He}$
}

\author{
S. Bacca ${ }^{1}$, N. Barnea ${ }^{2}$, W. Leidemann ${ }^{3}$, and G. Orlandini ${ }^{3, a}$ \\ 1 TRIUMF, 4004 Wesbrook Mall, Vancouver, B.C. V6J 2A3, Canada \\ 2 Racah Institute of Physics, Hebrew University, 91904, Jerusalem, Israel \\ 3 Dipartimento di Fisica, Università di Trento, and Istituto Nazionale di Fisica Nucleare, Gruppo Collegato di Trento, \\ I-38123 Trento, Italy
}

\begin{abstract}
In this contribution we summarize recent results on the longitudinal response function of ${ }^{4} \mathrm{He}$. It is intended to give an important contribution to one of the most interesting and much discussed topics in nuclear physics at present, i.e. the nuclear many-body forces. The longitudinal response is considered as a possible observable, involving many body scattering states, sensitive to the three-nucleon force (3NF). Such a sensitivity is predicted by ab initio calculations performed using the Lorentz Integral transform (LIT) method. The kinematics that are more interesting to measure are discussed.
\end{abstract}

\section{Introduction}

The nuclear potential has clearly an effective nature, therefore it is in principle a many-body operator. After several decades of theoretical and experimental studies of nucleonnucleon (NN) scattering observables the two-body part of this operator is rather well known. For the determination of a realistic three-body potential or to discriminate among different models one needs to find $\mathrm{A} \geq 3$ observables that are sensitive to it. One direction that has been followed [1, 2 ] is to calculate accurately bound properties of nuclei of increasing A. In fact it has been realized that stronger and stronger discrepancies exist between the binding energies calculated with high precision two-body potentials and the experimental values. Another very promising direction is to study electromagnetic reactions to states in the continuum. In fact many years of electron scattering experiments have demonstrated the power of this kind of reactions, and in particular of the inelastic ones, because of the possibility to vary energy $\omega$ and momentum $|\mathbf{q}|$ transferred by the electron to the nucleus. This allows one to focus on different dynamical aspects at different ranges and one might find regions where the searched three-body effects are sizable.

The ${ }^{4} \mathrm{He}$ nucleus is particularly appropriate for these studies because of the following considerations: i) the ratio between the number of triplets and of pairs goes like $(A-2) / 3$, therefore it is double for ${ }^{4} \mathrm{He}$ than for ${ }^{3} \mathrm{He}$; ii) theoretical results on hadron scattering observables involving four nucleons [3] as well as ${ }^{4} \mathrm{He}-\mathrm{N}$ phase shifts [4] seem to imply that three-body effects are rather large.

\section{Formalism}

For electron scattering reactions the inclusive differential cross section is given by

$$
\begin{aligned}
& \frac{d \sigma}{d \Omega_{e} d \omega}= \\
= & \sigma_{M}\left[\frac{Q^{4}}{|\mathbf{q}|^{4}} \sum_{n}|\langle n|\hat{\rho}| 0\rangle|^{2} \delta\left(\mathcal{E}_{n}-E_{0}-\omega\right)+\right. \\
+ & \left.\left(\frac{Q^{2}}{2|\mathbf{q}|^{2}}+\operatorname{tg}^{2} \frac{\theta}{2}\right) \sum_{n}\left|\left\langle n\left|\hat{\mathbf{J}}_{T}\right| 0\right\rangle\right|^{2} \delta\left(\mathcal{E}_{n}-E_{0}-\omega\right)\right] \\
\equiv & V_{L}(q, \omega) R_{L}+V_{T}(q, \omega, \theta) R_{T},
\end{aligned}
$$

where $\sigma_{M}$ denotes the Mott cross section, $Q^{2}=-q_{\mu}^{2}=$ $q^{2}-\omega^{2}$ the squared four momentum transfer with $\omega$ and $\mathbf{q}$ as energy and three-momentum transfers, respectively, and $\theta$ the electron scattering angle. The states $|0\rangle$ and $|n\rangle$ represent the initial (generally the ground state) and final states of the system, respectively. In the energy conserving $\delta$-function the final energy, represented by $\mathcal{E}_{n}$, includes both the internal energy of the system $E_{n}$ and the recoil energy acquired by the nucleus (non relativistically $E_{\text {rec }}=|\mathbf{q}|^{2} / 2 M_{A}^{2}$ with $M_{A}$ representing the mass of the nucleus).

The nuclear dynamics is in the so called response functions $R_{L}$ and $R_{T}$. They differ by the electromagnetic operators $\hat{\rho}$ and $\hat{\mathbf{J}}_{T}$ representing the nuclear charge and transverse current density operators, respectively. Their knowledge requires models about explicit and implicit degrees of freedom in the nuclear Hamiltonian. While the explicit ones (protons and neutrons) are obvious at the nuclear scale, the implicit ones are not. The latter are in fact the degrees of freedom underlying the potential model. For example for one boson exchange potentials they are represented by

\footnotetext{
a e-mail: orlandin@science.unitn.it
} 
the exchanged bosons whose charges and currents can couple to the electromagnetic field generated by the electrons. For phenomenological potentials they are unknown. It is clear that this uncertainty represents a problem if one wants to extract information on the potential, by comparing theory and experiment. Fortunately one can show [5] that the contributions to $\hat{\rho}$ and $\hat{\mathbf{J}}_{T}$ of subnuclear degrees of freedom appear at different relativistic orders. At lowest order the charge density operator contains only proton (and neutron if nucleons are thought as extended objects) degrees of freedom. Therefore the use of the simple one-body density operator

$$
\hat{\rho}(\mathbf{q})=\frac{e}{2} \sum_{k}\left(1+\tau_{k}^{3}\right) \exp \left[i \mathbf{q} \cdot \mathbf{r}_{k}\right]
$$

where $e$ is the proton charge and $\tau_{k}^{3}$ the isospin third component of nucleon $k$. allows to concentrate on the nuclear dynamics generated by the potential.

For the reasons exposed above we concentrate on the longitudinal response function $R_{L}$ at low energy and momentum transfer, where a consistent non relativistic quantum mechanical calculation can be performed. In particular, as the momentum transfer decreases we have the opportunity to see the evolution of the searched effects from the short range regime to the long range one.

It is well known that beyond the three-body break-up energies the calculation of scattering states for a four-body system is out of reach. We overcome the problem using the Lorentz Integral Transform (LIT) approach [6,7] which reduces the scattering problem to a bound state problem.

The LIT method consists in considering instead of $R_{L}$ an integral transform $\mathcal{L}_{L}(\sigma, q)$ with a Lorentzian kernel defined for a complex parameter $\sigma=\sigma_{R}+i \sigma_{I}$ by

$$
\mathcal{L}_{L}(\sigma, q)=\int d \omega \frac{R_{L}(\omega, q)}{\left(\omega-\sigma_{R}\right)^{2}+\sigma_{I}^{2}}=\left\langle\widetilde{\Psi}_{\sigma, q}^{\rho} \mid \widetilde{\Psi}_{\sigma, q}^{\rho}\right\rangle .
$$

The parameter $\sigma_{I}$ determines the resolution of the transform and is kept at a constant finite value $\left(\sigma_{I} \neq 0\right)$. The key point of the method is the theorem assuring that $\mathcal{L}_{L}$ can be evaluated from the norm of a function $\widetilde{\Psi}_{\sigma, q}^{\rho}$, that is the unique solution of the inhomogeneous equation

$$
\left(\hat{H}-E_{0}-\sigma\right)\left|\widetilde{\Psi}_{\sigma, q}^{\rho}\right\rangle=\hat{\rho}(q)\left|\Psi_{0}\right\rangle .
$$

Here $\hat{H}$ denotes the nuclear Hamiltonian. The existence of the integral in Eq. (3) implies that $\widetilde{\Psi}_{\sigma, q}^{\rho}$ has asymptotic boundary conditions similar to a bound state. Thus, one can apply bound-state techniques for its solution. The results presented here use the effective interaction hyperspherical harmonics $(\mathrm{EIHH})$ method $[8,9]$.

\section{Results and Discussion}

In the following results for the longitudinal response functions of ${ }^{4} \mathrm{He}$ are presented [10]. They are obtained using the the phenomenological Argonne V18 (AV18) NN potential [11] and two different 3NF, namely the Urbana IX (UIX) [12] and the Tucson-Melbourne (TM') [13].
In order to compare with experimental data both proton and neutron form factors have been included in the calculation, in particular the proton electric form factor has been described by the usual dipole parameterization

$$
G_{E}^{p}\left(Q^{2}\right)=G_{D}\left(Q^{2}\right)=\frac{1}{\left(1+\frac{Q^{2}}{\Lambda}\right)^{2}}
$$

$\left(\Lambda=18.43 \mathrm{fm}^{-2}\right.$ ) and the neutron electric form factor by the parameterization from [14].

Figure 1 shows the evolution of the $3 \mathrm{NF}$ effects on $R_{L}$ as the momentum transfer decreases. The effect at $q=50$ $\mathrm{Mev} / \mathrm{c}$ is especially large. Unfortunately no data are avail-
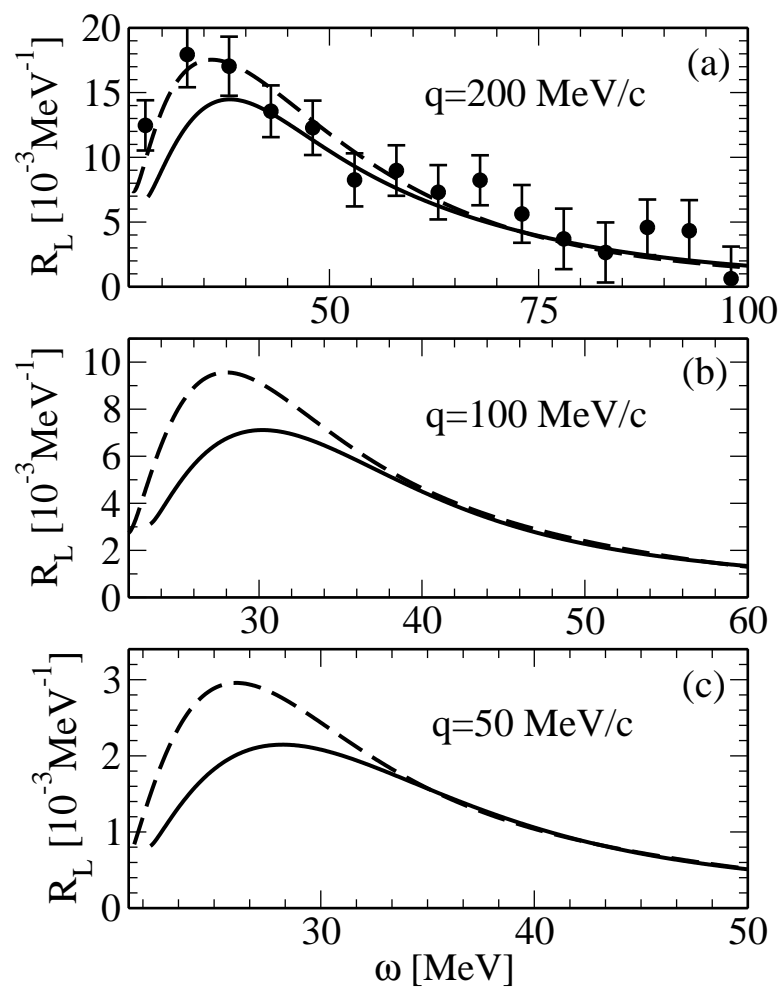

Fig. 1. $R_{L}(\omega, q)$ for $q=200,100$ and $50 \mathrm{MeV} / \mathrm{c}$ using AV18 only (dashed) and AV18+UIX (solid). Data from [15] .

able at the moment for the lowest $q$-values and the data at $q=200[15] \mathrm{MeV} / \mathrm{c}$ are too inaccurate to allow any conclusion.

The fact that $3 \mathrm{NF}$ effects are smaller for larger momentum transfer is confirmed in Fig. 2, (see also S. Bacca in these proceedings) where it is again clear that available data [16-18] do not have sufficient accuracy. However, Fig. 2 is interesting in that it shows that neglecting the final state interaction may have dramatic effects. In fact what is also shown in that figure with a dotted line is the so called quasi-elastic approximation to the response function. The approximation consists in assuming that the virtual photon interacts only with a single proton, which then leaves the nucleus undisturbed (plane wave). 


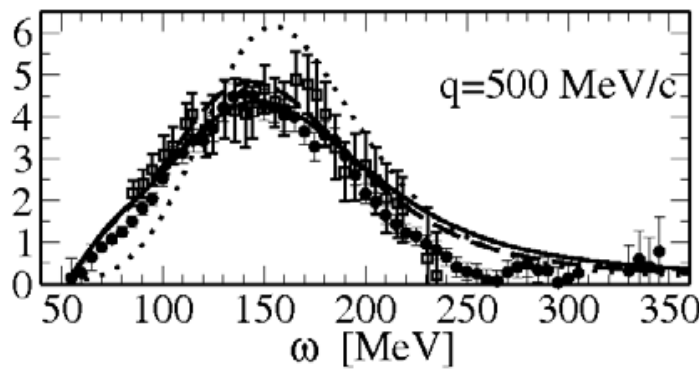

Fig. 2. $R_{L}(\omega, q)$ for $q=300,400$ and $500 \mathrm{MeV} / \mathrm{c}$ using AV18 only (dashed) and AV18+UIX (solid). The dotted line represents the quasi-elastic approximation. Data from [16] (squares), [17] (circles), [18] (open triangles).

Having found observables that show sizable effects of the UIX three-body potential one may wonder whether they can be used to discriminate between different $3 \mathrm{NF}$. To this aim we can compare the results in Fig. 1 with those obtained with the TM' potential [13]. The comparison is shown in Fig. 3.

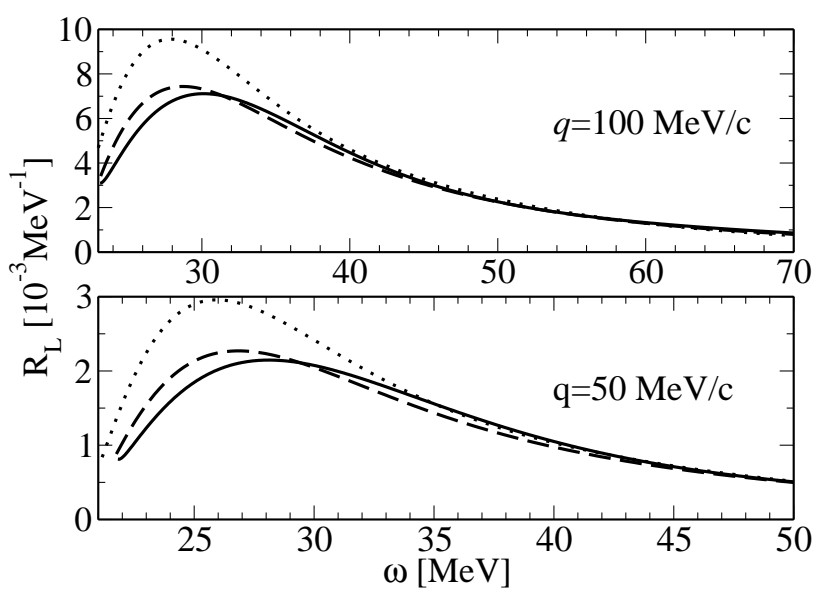

Fig. 3. $R_{L}(\omega, q)$ for $q=100$ and $50 \mathrm{MeV} / \mathrm{c}$ using AV18 only (dotted), AV18+UIX (solid) and AV18+TM (dashed).

Since the charge density operator in Eq. (2) has an isoscalar and an isovector part, the longitudinal response has been obtained calculating the isoscalar and isovector contributions separately. Moreover, each of them has been obtained as a sum of multipole contributions. It is interesting to investigate which multipole is most affected by the 3 NF. At first, however, it is instructive to see how the different multipoles contribute at different momentum transfer. To this aim in Figs. 4 and 5 we show how the isoscalar and isovector parts of $R_{L}$ are built up from their multipole contributions at a higher $(500 \mathrm{MeV} / \mathrm{c})$ and a lower $(100 \mathrm{MeV} / \mathrm{c})$ value of $q$. As expected, the higher the momentum transfer, the larger the number of multipoles that one has to consider to reach convergence. For $q=500$ $\mathrm{MeV} / \mathrm{c}$ up to seven multipoles are needed, while for $q=$
$100 \mathrm{MeV} / \mathrm{c}$ only three multipoles are required for a converged result. From the same figure one can infer that at higher $q$ the strength is almost equally distributed among the first isovector multipoles, while in the isoscalar channel the quadrupole gives the largest contribution. At low $q$, as expected, the response is dominated by the isovector dipole contribution, the isoscalar dipole is completely negligible and the isoscalar quadrupole contributes a few percent. Negligible strength is found for the multipoles higher than the quadrupole (similar to the isoscalar dipole case).

Coming to the $3 \mathrm{NF}$ effects, in Fig. 6 the dominating dipole contribution to the longitudinal response function is shown. On can notice that the effect of the $3 \mathrm{NF}$ is very similar to that in Fig. 1(b). This is due both to the fact that this is the dominant contribution and to some cancellations of the $3 \mathrm{NF}$ contributions to the other multipoles.
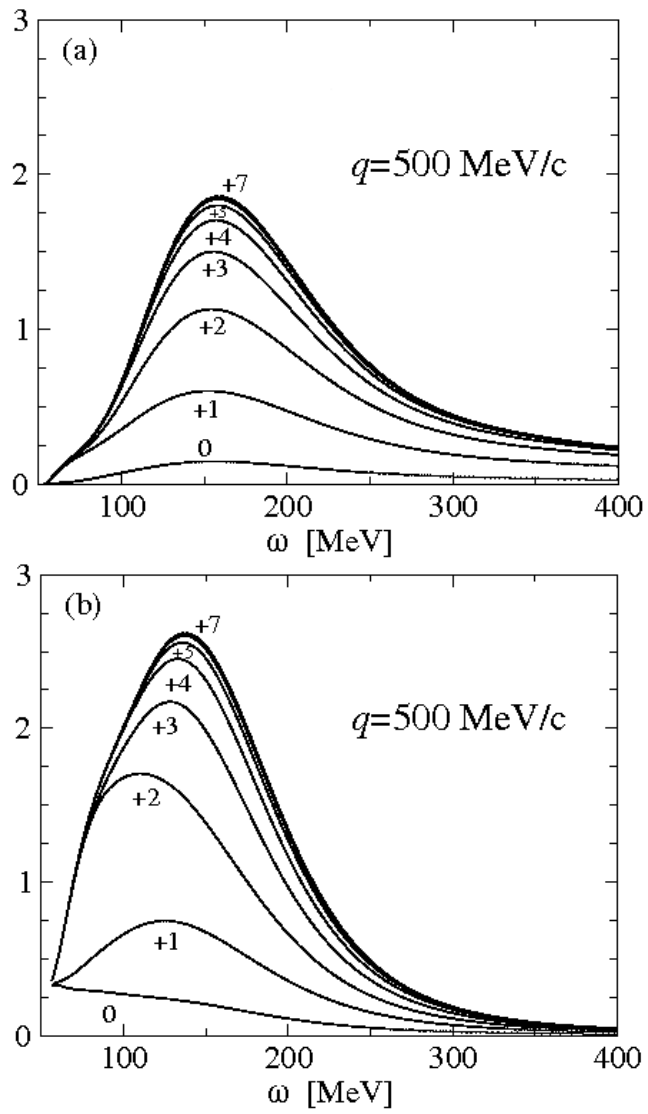

Fig. 4. (a) and (b): isovector and isoscalar response functions, respectively, built up from the lowest $J$ multipole contributions for $q=500 \mathrm{MeV} / \mathrm{c}$. The results are obtained with the AV18+UIX potential.

Finally we want to discuss one of the most interesting integral properties of the longitudinal response function, i.e. the so called Coulomb Sum Rule (CSR) (for a review see $[19,20])$. The main interest in this sum rule comes from its connection to the Fourier transform $\left(f_{p p}(q)\right)$ of the proton-proton correlation function $\left(\rho_{p p}(s)\right)$, i.e the probability to find two protons at a distance $s$. In fact one has 

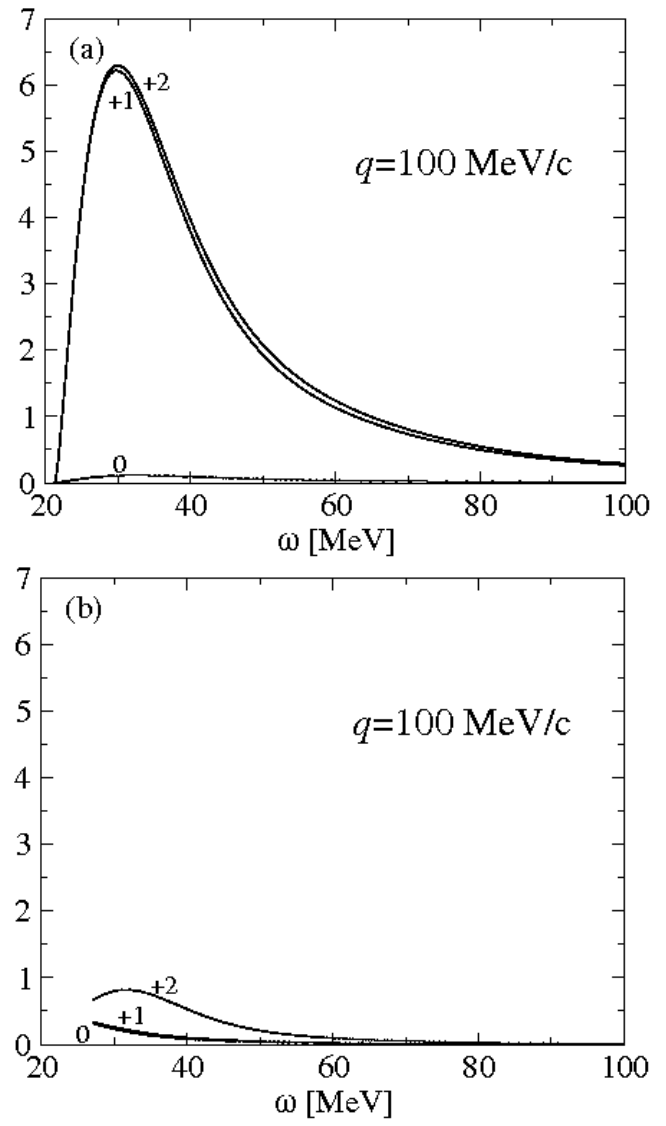

Fig. 5. The same as Fig. 4, but for $q=100 \mathrm{MeV} / \mathrm{c}$.

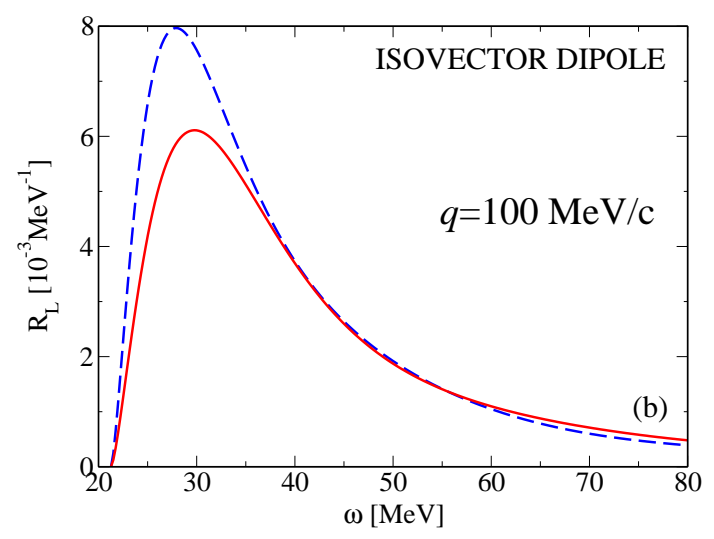

Fig. 6. Response function of the isovector dipole for $q=$ $100 \mathrm{MeV} / \mathrm{c}$ with the AV18 (dashed) and AV18+UIX (solid) potentials.

$\operatorname{CSR}(q) \equiv \int_{\omega_{t h}} d \omega R_{L}(\omega, q)=Z+Z(Z-1) f_{p p}(q)-Z^{2}|F(q)|^{2}$,

where $F(q)$ is the nuclear elastic form factor. Therefore it is interesting to see what is the effect of the $3 \mathrm{NF}$ on $f_{p p}(q)$. This is shown in Fig. 7. One can see that the effect increases up to $25 \%$ at $q=400 \mathrm{MeV} / \mathrm{c}$. However, in view of the fact that Eq. (6) has been obtained with the operator

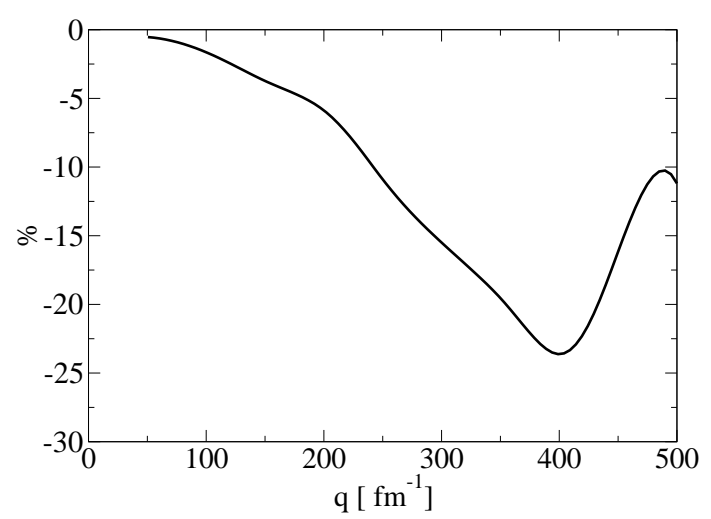

Fig. 7. Percent difference between $f_{p p}(q)$ calculated with AV18 only and AV18+UIX.

in Eq. (2), which neglects contributions of higher relativistic order, one has to keep in mind that only kinematics at $q \lesssim 300 \mathrm{MeV} / \mathrm{c}$, where the effect amounts up to $15 \%$, can be considered safe for a possible comparison with experimental data, giving also an idea of the required experimental accuracy.

\section{Conclusions}

In this contribution we have shown that the longitudinal response function of ${ }^{4} \mathrm{He}$ is potentially a crucial observable for the search of $3 \mathrm{NF}$ effects. In fact at the lowest momentum transfers investigated (50-100 MeV/c) the comparison of the results obtained with and without $3 \mathrm{NF}$ shows large effects of the $3 \mathrm{NF}$, up to $30-40 \%$. Moreover, using two phenomenological potentials one finds slightly shifted peaks. The result is a difference between the two responses that can be as large as $10-15 \%$. The multipole analysis shows that the largest $3 \mathrm{NF}$ effect is just on the dominating isovector dipole.

Three-body force effects are visible in the Coulomb sum rule as well, i.e. on the Fourier transform of the protonproton correlation function. Three-body correlations due to the potential reach the maximum around 300-400 MeV/c. This could reflect the intermediate-long range nature of the 3NF.

At present experimental data are available for $q \geq 200$ $\mathrm{MeV} / \mathrm{c}$, where the $3 \mathrm{NF}$ effects are smaller and are not sufficiently precise to draw any conclusion. A Rosenbluth separation of the inclusive electron scattering cross section of ${ }^{4} \mathrm{He}$ at momentum transfer $q \leq 200 \mathrm{MeV} / \mathrm{c}$ would be of high value in view of a more accurate determination of the three-body force and in general of the intermediate-long range dynamics of this system.

\section{References}

1. Steven C. Pieper, R. B. Wiringa, and J. Carlson, Phys. Rev. C70, (2004) 054325 
2. R. B. Wiringa, Steven C. Pieper, J. Carlson, and V. R. Pandharipande, Phys. Rev. C62, (2000) 014001

3. A. Deltuva, A.C. Fonseca, and P.U. Sauer, Phys. Lett. B660, (2008) 471

4. K.M. Nollett, S.C. Pieper, R.B. Wiringa, J. Carlson, and G.M. Hale, Phys. Rev. Lett. 99, (2007) 022502

5. L.L. Foldy and S.A. Wouthuysen, Phys. Rev. 78, (1950) 29

6. V.D. Efros, W. Leidemann, and G. Orlandini, Phys. Lett. B338, (1994) 130

7. V.D. Efros, W. Leidemann, G. Orlandini, and N. Barnea, J. Phys. G: Nucl. Part. Phys. 34, (2007) R459

8. N. Barnea, W. Leidemann, and G. Orlandini, Phys. Rev. C61, (2000) 054001; Nucl. Phys. A 693,(2001) 565

9. N. Barnea, V. D. Efros, W. Leidemann, and G. Orlandini, Few-Body Syst. 35, (2004) 155

10. S. Bacca, N. Barnea, W. Leidemann, and G. Orlandini, Phys. Rev. Lett. 102, (2009) 162501; Phys. Rev. C 80, (2009) 064001

11. R.B. Wiringa, V.G.J. Stoks, and R. Schiavilla, Phys. Rev. C C51, (1995) 38

12. B. S. Pudliner and V. R. Pandharipande, J. Carlson, S. C. Pieper, and R. B. Wiringa, Phys. Rev. C56, (1997) 1720

13. S. A. Coon and H. K. Hahn, Few-Body Syst. 30, (2001) 131

14. S. Galster et al., Nucl. Phys. B32, (1971) 221

15. A. Yu. Buki, I. S. Timchenko, N. G. Shevchenko, and I. A. Nenko, Phys. Lett. B641, (2006) 156

16. S. A. Dytman et al., Phys. Rev. C38, (1988) 800

17. A. Zghiche et al., Nucl. Phys. A572, (1994) 513

18. J. Carlson, J. Jourdan, R. Schiavilla, and I. Sick, Phys. Rev. C65, (2002) 024002

19. G. Orlandini and M. Traini, Rep. Prog. Phys. 54, (1991) 257

20. O. Benhar, D. Day, and I. Sick, Rev. Mod. Phys. 80, (2008) 189 